\title{
An Evaluation of Diagnosis-Related Group (DRG) Implementation Focused on Cancer DRGs in Greek Public Hospitals
}

\author{
Panos Panagiotopoulos $^{1}\left[\right.$ [D $\cdot$ Nikos Maniadakis ${ }^{2} \cdot$ George Papatheodoridis $^{1} \cdot$ Dimitris Pektasidis $^{1}$
}

Published online: 20 May 2019

(c) The Author(s) 2019

\begin{abstract}
Objectives The main aims of this study were to evaluate the Greek version of the diagnosis-related group reimbursement system (KEN-DRG) and to compare the KEN-DRG prices with the average actual cost of each group of study cases. Along with other aspects, the differences between the KEN-DRG average length of stay (ALOS) and the actual ALOS was evaluated in selected cases.

Methods In the first part of this study, the top-down costing approach was selected in order to break down the total operating costs of the hospital, by hospital department. The aim of this stage was identification of the total operating costs and the average cost per patient day for each Internal Medicine Department of the 'Hippokration' General Hospital of Athens during the period 2014-2015. The final cost drivers were identified using the concept of cluster-related incidents in the hospital. In a subsequent stage, the 13 most frequent cancer KEN-DRG prices charged by Internal Medicine Departments were selected as a sample for further data analysis.

Results With regard to the costing of the oncological KEN-DRG, the present study illustrates that a majority of the current reimbursement rates for oncological KEN-DRG codes are under-reimbursed, taking into account the actual costs of hospitalization for each group of cases. The results also reveal that the ALOS of the KEN-DRG does not reflect the actual ALOS in the sample of cases examined. In addition, under the scope of this study, two proposed models for the KEN-DRG price recalculation were developed, based on the average estimated cost of hospitalization for the sample incidents, which could improve the existing reimbursement system for Greek hospitals in the medium term.

Conclusions The KEN-DRG payment system that was implemented in Greece for the first time in 2012 needs redesigning in terms of the true cost of hospital services and the actual cost of each patient's treatment. With regard to the existing KENDRG reimbursement system, the current study suggested the use of a DRG price calculation model that consists of a relative weight factor and a base price, based on a real cost calculation process on an annual basis. Moreover, it should be stressed that the present study, as well as other related studies, make it possible to know the actual cost of hospitalization, and can contribute to the creation of a cost database over time at the level of hospitals or specific clinical departments.
\end{abstract}

\section{Introduction}

Diagnosis-related groups (DRGs) are part of an internationally recognized system for hospital reimbursement/funding based on the classification of patients into groups according to the costs consumed during their hospitalization, which depend on their clinical characteristics and the services provided to them [7].

Panos Panagiotopoulos

panapa13@otenet.gr

1 Medical School, National and Kapodistrian University of Athens (UOA), Athens, Greece

2 National School of Public Health (NSPH), Athens, Greece

\section{Key Points for Decision Makers}

The evaluation of cancer diagnosis-related groups (DRGs) revealed significant payment disparities between the actual costs of hospitalization and the hospital reimbursement amount.

The use of a new DRG price calculation model is proposed that consists of a relative weighting factor and a base price, based on a real cost calculation process on an annual basis.

The launch of the Greek DRG system triggered the need for further research in order for the DRG model to fulfill its promise. 
DRGs were first used in the US in 1983 as a compensation scheme for the Medicare health program (Federal Health Care Program for the elderly and disabled).

Since then they have been adopted, with local variations, by the health care systems of many developed countries [1, $6]$.

Among the European countries, Portugal first applied DRGs in 1988, followed by Norway in 1991-1993 in selected hospitals, and Ireland in 1993. Within a short period of time, the DRG system has spread to many European countries. Most of these countries, including Germany, France, Finland, Estonia and others, adopted the DRG system that had already been applied in the US and Australia and used it as a basis for further developing their own systems. In contrast, England and Austria preferred to create a new system of similar diagnostic categories from scratch, while Poland used the English version as the basis for its own system [3, 7].

The reasons why all these countries chose to apply DRGs could be summarized into two main categories. The first category includes goals such as reducing hospital costs, motivating the efficient use of resources in hospitals, and thus increasing efficiency, while the second category mainly involves increasing the transparency of the services provided. It was thought that the combination of these reasons would contribute to an increase in the quality of care [2, 9].

In Greece, until 2011, the retrospective payments system was applied: a reimbursement mechanism that was considered inflationary and was held responsible for a series of problems, such as high administrative costs, a lack of control of hospital expenses, an increase in the average duration of hospitalization and the creation of deficits [15].

By the end of 2011, the need for the Greek health care system to reform under the pressure of the financial crisis, augmented by the Greek government's commitment to other member states of the European Stability Mechanism to implement a number of measures, led to the implementation of a prospective reimbursement mechanism for hospitalsthe Greek version of the DRG system, called KEN-DRG [5].

Within the framework of the first economic adjustment program for Greece, the Ministry of Health estimated that the implementation of the KEN-DRG system would yield great benefits in terms of management time and cost, for both hospitals and insurance organizations. In particular, the main objective of the KEN-DRG implementation in Greece was cost control through a reduction in the funding of public hospitals from the state budget, as well as an increase in transparency and an improvement in the overall efficiency of hospitals [8].

At the end of 2011, the Greek Ministry of Health, together with a group of scientists specializing in Health Economics, decided that the best solution was to adapt the Australian DRG system (AR-DRG, 6th edition) to Greek data. The reason for this choice was that, among the available models, the AR-DRG had found wider acceptance according to the international literature, and had already been adopted successfully by many countries.

A scientific committee was set up by the Health Ministry to translate the AR-DRG code list into Greek, making any necessary modifications, and to develop proposals for a quick transformation and implementation of the system, taking into account the strengths, weaknesses and priorities of the Greek social security and health care system. In collaboration with experts, after conducting microcosting techniques on selected cases from Greek public hospitals, the committee delivered a less sophisticated model than the AR-DRG, based on AR-DRG cost-weight tables and the average cost of the selected cases [8].

In this context, the first Greek version of the DRG system (KEN-DRG) was a generalized hospital price list of 700 medical reimbursement packages (grouped together into 25 major diagnostic categories) that determines the cost and average length of stay (ALOS) for each DRG of patients. These data theoretically corresponded to the averages for each category of patients and are used for the direct charging of hospital incidents to the insurers, regardless of the individual charges and the actual cost of hospitalization [8].

The classification of patients' incidents using KEN-DRG codes is based on the diagnoses describing the patient's condition (International Classification of Diseases, Tenth Revision [ICD-10] codes), as well as on the surgical procedures and health interventions performed [Greek coding of medical procedures (ELOKIP) codes]. In terms of completeness of mappings, $81 \%$ of KEN-DRGs were assigned to ICD-10 codes, whereas the corresponding rate of correlation with surgical procedures and health intervention codes was lower than $65 \%$ [12].

In March 2012, the latest major changes were made in the KEN-DRG system, with adjustments to pricing, coding, ALOS and number of categories, leading to a revised list of 760 hospital reimbursement packages. From that point until today, only a few minor adjustments have been made to the KEN-DRG system. However, an analysis of the KEN-DRG data, carried out in the first years of implementation, found that $8-21 \%$ of total hospital revenue resulted from extreme payment prices (outliers). This meant that the current system required corrective modifications; to date, four revisions have been made [11].

Another important weakness in the implementation of KEN-DRG in Greece was that the specialized software (grouper) for the classification of incidents into appropriate KEN-DRGs was not acquired by the Greek government. An application was developed by the Ministry of Health for this purpose but it had significant deficiencies, with the result that patient billing offices rank incidents manually in diagnostic categories based on their own judgement. 
The aim of this research was to evaluate KEN-DRG and to compare its prices with the actual average cost of each group of study cases. Along with other aspects, the difference between the KEN-DRG ALOS and the actual ALOS of selected cases was evaluated.

\section{Materials and Methods}

The data used in this research were collected from the 'Hippokration' General Hospital of Athens during 2014 and 2015. In the context of this study, the hospital's Finance Department provided a database containing all the financial data for the above period. More analytically, accounting data sheets and records were analyzed in order to allocate actual total operating costs to the hospital's Internal Medicine Departments. The research population for this study included all hospitalizations in those departments for the years 2014 and 2015, equating to 3750 and 4045, respectively.

The data extracted from the hospital financial record database included accounting data sheets as well as reimbursement administrative data for the total patient population. These contained patient demographic data such as sex and age, length of stay, name and code of clinic, total billing amount, KEN-DRG billing code and price, and other hospital charges. All data were anonymized with respect to patient identification.

In the first stage of the research, the top-down costing approach was selected in order to break down costs by hospital department. In this costing procedure, the first step was to apportion the overheads to hospital services, while the second step was to apportion the costs of secondary cost centers to primary cost centers on an equitable basis. The main aim of this stage was the identification of the total operating costs and the average cost per patient day for each Internal Medicine Department of the 'Hippokration' General Hospital during 2014-2015.

The next procedure required within the framework of this methodological approach was the selection of patients who would make up the targeted sample of cases for the case-study analysis stage. The basic criterion for the selection of samples for these particular hospital units was their specialization in the treatment of different types of cancer. With regard to this criterion, the targeted sample of hospitalization cases consisted only of oncological incidents that were charged with a KEN-DRG that belonged mainly to the 17th Main Diagnostic Category (MDC 17) in the 'Neoplastic disorders (hematological and solid neoplasms)' group. The 13 most frequent oncological KEN-DRGs were selected as a sample, based on the total population data for the years 2014 and 2015. In addition, in order to avoid the influence of extreme values on the selected sample and to eliminate the consequences of non-regularity of the errors, it was decided to reject (as outliers) the values that were three times greater or less than the average value. In particular, a total of 43 cases with extreme values in terms of length of stay and cost were rejected from the selected sample (558 cases), resulting in a total sample of 515 incidents.

\section{Results}

\subsection{First Stage: Cost Estimation for Internal Medicine Departments}

In order to calculate the total cost of the services offered by the two Internal Medicine Departments of the 'Hippokration' General Hospital of Athens, it was necessary to define the main and auxiliary cost centres. The costing of services for these clinics refers to the determination of the total cost of hospitalization cases in terms of medicines and medical supplies, nursing and medical care, and those overheads allocated to nursing departments as necessary for the overall functioning of the hospital. The main results of this cost calculation revealed that the total cost for both Internal Medicine Departments for the years 2014 and 2015 was $€ 15,941,380$, while the average daily cost of hospitalization was $€ 368.28$.

For the purposes of this study, the reimbursement data of the cases hospitalized in the two Internal Medicine Departments during 2014-2015 were divided into two main reimbursement methods: cases charged using KEN-DRG reimbursement, and cases charged using fee-for-service.

Based on the KEN-DRG coding system defined by the Ministry of Health, the fourth letter of the KEN-DRG code has the following meaning: $\mathrm{M}=$ with complications; $\mathrm{X}=$ without complications; $\mathrm{A}=$ no other indication.

Considering all of the above, in order to get as close as possible to the real cost of hospitalization per incident, we created a weighting factor for the average daily cost of hospitalization, based on the following two main categories of incident: (1) fee-for-service pricing (this category mainly includes short-term and low-cost cases) or KEN-DRG pricing with a code whose fourth letter was A (no other indication) or X (no complications); (2) KEN-DRG pricing with a code whose fourth letter was $\mathrm{M}$ (with complications). Both the total and average daily hospitalization costs were then recalculated for each category (Table 1 ).

\subsection{Second Stage: Reimbursement Data Analysis of the Selected Sample}

Based on the selected sample of the most common oncological cases, the most frequent KEN-DRG code charged was $\Pi 40 X$ (Digestive Malignancy W/O Catastrophic CC), 
Table 1 Weighting of the average cost per patient day (total number of pathology clinic cases, 2014-2015)

\begin{tabular}{|c|c|c|c|}
\hline & \multicolumn{2}{|l|}{ KEN-DRG weight categories } & \multirow[t]{3}{*}{ Total } \\
\hline & $\mathrm{A}$ & $\mathrm{B}$ & \\
\hline & $\begin{array}{l}\text { Cases with and without } \\
\text { KEN-DRG codes A and X }\end{array}$ & $\begin{array}{l}\text { Cases with KEN- } \\
\text { DRG code M }\end{array}$ & \\
\hline Number of cases & 6331 & 1464 & 7795 \\
\hline Average length of stay, days & 4.1 & 12.0 & 5.6 \\
\hline Total hospitalization days & 25,781 & 17,505 & 43,286 \\
\hline Average cost per patient day & $€ 368.28$ & $€ 368.28$ & $€ 368.28$ \\
\hline Total cost per category & $€ 9,494,634$ & $€ 6,446,746$ & $€ 15,941,380$ \\
\hline Weight coefficient & 0.9 & 1.1 & \\
\hline Recalculation of total cost per category & $€ 8,767,759$ & $€ 7,173,621$ & $€ 15,941,380$ \\
\hline Weighted average cost per patient day & $€ 340.09$ & $€ 409.80$ & $€ 368.28$ \\
\hline $\begin{array}{l}\text { Weighted average cost per hospitalization } \\
\text { case (base rate) }\end{array}$ & $€ 1384.89$ & $€ 4900.01$ & $€ 2045.08$ \\
\hline
\end{tabular}

$K E N-D R G$ Greek diagnosis-related group followed by $\Sigma 20 \mathrm{M}$ (Acute Leukemia W Catastrophic CC) and $\Sigma 21 \mathrm{M}$ (Lymphoma and Non-Acute Leukemia W Catastrophic CC).

In a next step, in order to derive useful conclusions regarding the KEN-DRG billing code of the sample cases, the actual ALOS of the associated hospital cases was compared with the corresponding ALOS of KEN-DRG. In the vast majority of cases, the actual ALOS exceeded the KENDRG ALOS. Higher excess percentages occurred in hospital cases with KEN-DRG billing codes equal to $\Sigma 20 \mathrm{M}$ (Acute Leukemia W Catastrophic CC; 80\%), s01 M (Lymphoma and Leukemia W Major OR Procedures W Catastrophic or Severe CC; 68\%), Г20X (Malignancy, Female Reproductive System W/O Catastrophic CC; 58\%), ऽ21M (Lymphoma and Non-Acute Leukemia W Catastrophic CC; 55\%) and ח40X (Digestive Malignancy W/O Catastrophic CC; 53\%).

In addition, it was considered important to investigate the level of excess of the actual average hospital reimbursement amount in relation to the cancer KEN-DRG code prices. For KEN-DRG codes $\Sigma 22 \mathrm{X}$ (Other Neoplastic Disorders W/O CC; excess of 63\%), A31M (Respiratory Neoplasms W Catastrophic CC; excess of 42\%), П40X (Digestive Malignancy W/O Catastrophic CC; excess of $41 \%$ ) and $\Sigma 20 \mathrm{M}$ (Acute Leukemia W Catastrophic CC; excess of 33\%), the average hospital reimbursement amount significantly exceeded the KEN-DRG price.

A reasonable question arises as to whether there was an interaction between the hospital charges and the length of stay in the selected cases. The analysis revealed a strong positive correlation between these two variables $(r=0.91)$. Accordingly, we can conclude that when the predefined ALOS of cancer KEN-DRG codes is exceeded by the actual average ALOS of hospital cases, there is also an excess of the average hospital reimbursement amount in relation to the KEN-DRG price.

\subsection{Third Stage: Price Evaluation of the KEN-DRG Reimbursement Method}

In order to answer one of the key questions of the survey, i.e. whether or not the price assigned to each KEN-DRG code by the Greek Ministry of Health corresponds to the actual cost of hospitalization, we compared the average estimated cost of hospitalization (costing analysis) with the predetermined price that corresponded to each of the examined cancer KEN-DRGs. More specifically, the average estimated cost of hospitalization for each KEN-DRG code examined was calculated by multiplying the average daily cost of hospitalization, taking into account the code weighting factor described above, by the ALOS associated with that code (Table 2).

As can be seen from the table, the estimated average cost of hospitalization resulting from the above calculations differed significantly from the actual average hospital reimbursement amount for the corresponding KEN-DRG.

More specifically, the greatest amount by which the estimated average cost of hospitalization exceeded the average reimbursement was observed for the KEN-DRG with code A31X (Respiratory Neoplasms W/O Catastrophic CC; excess of $205 \%$ ). Excesses of more than $100 \%$ of the reimbursement amount were also observed for codes П40 M (Digestive Malignancy W Catastrophic CC; excess of 178\%), Y22 M (Kidney and Urinary Tract Neoplasms W Catastrophic or Severe CC; excess of 139\%), A31 M (Respiratory Neoplasms W Catastrophic CC; excess of 126\%) and $\Pi 40 X$ (Digestive Malignancy W/O Catastrophic CC; excess of $103 \%$ ). Smaller deviations from the average reimbursement amount were seen for codes $\Sigma 21 \mathrm{X}$ (Lymphoma and Non-Acute Leukemia W/O Catastrophic CC; excess of - 4\%), $501 \mathrm{M}$ (Lymphoma and Leukemia W Major OR Procedures W Catastrophic or Severe CC; excess of $31 \%$ ), 
Table 2 Calculation of average estimated cost per case on the basis of average real length of stay

\begin{tabular}{|c|c|c|c|c|c|c|c|}
\hline $\mathrm{R} / \mathrm{N}$ & $\begin{array}{l}\text { KEN-DRG } \\
\text { code }\end{array}$ & KEN-DRG description & $\begin{array}{l}\text { KEN-DRG } \\
\text { price }\end{array}$ & $\begin{array}{l}\text { Average actual } \\
\text { LOS by KEN- } \\
\text { DRG (days) }\end{array}$ & $\begin{array}{l}\text { Average actual } \\
\text { total reimburse- } \\
\text { ment amount } \\
\text { (hospital charges) }\end{array}$ & $\begin{array}{l}\text { Average estimated } \\
\text { cost per patient } \\
\text { day (cost analysis) }\end{array}$ & $\begin{array}{l}\text { Estimated average } \\
\text { cost per hospitali- } \\
\text { zation case based } \\
\text { on average actual } \\
\text { LOS }\end{array}$ \\
\hline 1 & $\Pi 40 \mathrm{X}$ & $\begin{array}{l}\text { Digestive Malignancy W/O } \\
\text { Catastrophic CC }\end{array}$ & $€ 600$ & 4.5 & $€ 748$ & $€ 340$ & $€ 1520$ \\
\hline 2 & $\Sigma 20 \mathrm{M}$ & $\begin{array}{l}\text { Acute Leukaemia W Cata- } \\
\text { strophic CC }\end{array}$ & $€ 5707$ & 27.4 & $€ 8020$ & $€ 410$ & $€ 11,225$ \\
\hline 3 & $\Sigma 21 \mathrm{M}$ & $\begin{array}{l}\text { Lymphoma and Non-Acute } \\
\text { Leukaemia W Catastrophic } \\
\text { CC }\end{array}$ & $€ 3100$ & 17 & $€ 4135$ & $€ 410$ & $€ 6971$ \\
\hline 4 & $\Sigma 21 \mathrm{X}$ & $\begin{array}{l}\text { Lymphoma and Non-Acute } \\
\text { Leukaemia W/O Cata- } \\
\text { strophic CC }\end{array}$ & $€ 1410$ & 4.3 & $€ 1519$ & $€ 340$ & $€ 1462$ \\
\hline 5 & $\mathrm{~A} 31 \mathrm{X}$ & $\begin{array}{l}\text { Respiratory Neoplasms W/O } \\
\text { Catastrophic CC }\end{array}$ & $€ 828$ & 8.3 & $€ 926$ & $€ 340$ & $€ 2826$ \\
\hline 6 & $\mathrm{H} 41 \mathrm{X}$ & $\begin{array}{l}\text { Malignancy of Hepatobiliary } \\
\text { System, Pancreas W/O } \\
\text { Catastrophic CC }\end{array}$ & $€ 792$ & 4 & $€ 927$ & $€ 340$ & $€ 1350$ \\
\hline 7 & $\mathrm{H} 41 \mathrm{M}$ & $\begin{array}{l}\text { Malignancy of Hepatobiliary } \\
\text { System, Pancreas W Cata- } \\
\text { strophic CC }\end{array}$ & $€ 1754$ & 8 & $€ 1913$ & $€ 410$ & $€ 3266$ \\
\hline 8 & $\Sigma 22 \mathrm{X}$ & $\begin{array}{l}\text { Other Neoplastic Disorders } \\
\text { W/O CC }\end{array}$ & $€ 638$ & 3 & $€ 751$ & $€ 340$ & $€ 1020$ \\
\hline 9 & $\Pi 40 \mathrm{M}$ & $\begin{array}{l}\text { Digestive Malignancy W } \\
\text { Catastrophic CC }\end{array}$ & $€ 1100$ & 12.1 & $€ 1791$ & $€ 410$ & $€ 4971$ \\
\hline 10 & $\mathrm{~A} 31 \mathrm{M}$ & $\begin{array}{l}\text { Respiratory Neoplasms W } \\
\text { Catastrophic CC }\end{array}$ & $€ 1634$ & 10.7 & $€ 1933$ & $€ 410$ & $€ 4369$ \\
\hline 11 & $\Upsilon 22 \mathrm{M}$ & $\begin{array}{l}\text { Kidney and Urinary Tract } \\
\text { Neoplasms W Catastrophic } \\
\text { or Severe CC }\end{array}$ & $€ 1373$ & 11.4 & $€ 1952$ & $€ 410$ & $€ 4672$ \\
\hline 12 & $\Sigma 01 \mathrm{M}$ & $\begin{array}{l}\text { Lymphoma and Leukaemia } \\
\text { W Major OR Procedures W } \\
\text { Catastrophic or Severe CC }\end{array}$ & $€ 8000$ & 31.2 & $€ 9788$ & $€ 410$ & $€ 12,790$ \\
\hline 13 & Г20X & $\begin{array}{l}\text { Malignancy, Female } \\
\text { Reproductive System W/O } \\
\text { Catastrophic CC }\end{array}$ & $€ 1100$ & 5.4 & $€ 1250$ & $€ 340$ & $€ 1843$ \\
\hline
\end{tabular}

$C C$ Catastrophic or Severe CC, $K E N-D R G$ Greek diagnosis-related group, $L O S$ length of stay, $R / N$ row number, $W$ with, $W / O$ without

$\Sigma 22 \mathrm{X}$ (Other Neoplastic Disorders W/O CC; excess of 36\%), इ20M (Acute Leukemia W Catastrophic CC; (excess of 40\%) and H41X (Malignancy of Hepatobiliary System, Pancreas W/O Catastrophic CC; excess of 46\%). Despite the fact that the hospital payroll is not included in KEN-DRG prices, and accounts for approximately $40 \%$ of the total hospital costs, the above results reveal that the current prices of cancer KEN-DRG codes are seriously underestimated in relation to the actual costs of hospitalization.

On the basis of the above comparison, the greatest deviations of the average estimated cost per hospitalization from the average KEN-DRG-based reimbursement amount were observed in KEN-DRG codes with a fourth letter code of M (with complications), i.e. in severe cases, with the exception of the KEN-DRG code A31X (Respiratory Neoplasms W/O Catastrophic CC).

\section{Proposed/Estimated Models}

Given the discrepancies between KEN-DRG pricing and the actual costs, two proposed models for modification of the KEN-DRG system were developed, based on the average estimated cost of hospitalization of the sample incidents, which could improve the existing reimbursement system for Greek hospitals in the medium term. At the same time, the 
proposed models could serve as a basis for global budget implementation in Greek public hospitals.

The proposed modification models, as in most DRG systems successfully implemented by different countries, incorporate a factor that indicates the relative weight in terms of resource consumption and consequent cost of each DRG. In addition, in order to determine the price of a DRG, it is necessary to calculate the base price, which is the average amount reimbursed per incident for all hospitals in the country on an annual basis. Subsequently, the determination of the reimbursement amount of each DRG is derived by multiplying the base price by the relative weight of the DRG.

As the base price for the implementation of proposed models, we defined the average cost of hospitalization as $€ 2045.08$, as calculated from the first stage of analysis for the Internal Medicine Departments of the 'Hippokration' General Hospital.

\subsection{Proposal A}

In Table 3, the basic parameters of the proposed model A are analyzed in detail for each of the cancer KEN-DRGs examined in this study. Column 7 of the table illustrates the relative weighting coefficient per DRG resulting from the quotient of the actual average cost per KEN-DRG, based on its actual ALOS divided by the calculated base price. The last column indicates the proposed DRG price, which is the actual average cost for the corresponding KEN-DRG, as calculated based on the actual average duration of hospitalization for the sample incidents.

\subsection{Proposal B}

In the context of this study, and considering that the initial coding of the KEN-DRG was based on version 6.0 of the AR-DRG patient classification system, with a number of modifications and interventions, we correlated the codes of the tested cancer KEN-DRGs with the corresponding ARDRG classification codes of the above version. In this alternative proposal for calculating the proposed DRG price for each of the examined KEN-DRGs (Column 10 of Table 4), the actual average cost of hospitalization of the incidents treated in the two departments studied for the years 2014 and 2015 was used as a base rate, while the relative weighting coefficients of the AR-DRG system codes were used as the relative weights for the DRG price calculation.

Table 3 Prediction model of DRG price based on the examined KEN-DRG: Proposal A

\begin{tabular}{|c|c|c|c|c|c|c|c|}
\hline 1 & 2 & 3 & 4 & 5 & 6 & 7 & 8 \\
\hline $\mathrm{R} / \mathrm{N}$ & KEN-DRG code & KEN-DRG description & $\begin{array}{l}\text { Length of stay } \\
\text { by KEN-DRG }\end{array}$ & KEN-DRG price & Base price & $\begin{array}{l}\text { Proposed } \\
\text { relative } \\
\text { weights }\end{array}$ & $\begin{array}{l}\text { Proposed } \\
\text { DRG price }\end{array}$ \\
\hline 1 & $\Pi 40 \mathrm{X}$ & Digestive malignancy W/O catastrophic CC & 3 & $€ 600$ & $€ 2045$ & 0.74 & $€ 1520$ \\
\hline 2 & $\Sigma 20 \mathrm{M}$ & Acute leukaemia W catastrophic CC & 17 & $€ 5707$ & $€ 2045$ & 5.49 & $€ 11,225$ \\
\hline 3 & $\Sigma 21 \mathrm{M}$ & $\begin{array}{l}\text { Lymphoma and non-acute leukaemia W } \\
\text { catastrophic CC }\end{array}$ & 14 & $€ 3100$ & $€ 2045$ & 3.41 & $€ 6971$ \\
\hline 4 & $\Sigma 21 \mathrm{X}$ & $\begin{array}{l}\text { Lymphoma and non-acute leukaemia W/O } \\
\text { catastrophic CC }\end{array}$ & 5 & $€ 1410$ & $€ 2045$ & 0.72 & $€ 1462$ \\
\hline 5 & $\mathrm{~A} 31 \mathrm{X}$ & $\begin{array}{l}\text { Respiratory neoplasms W/O catastrophic } \\
\text { CC }\end{array}$ & 5 & $€ 828$ & $€ 2045$ & 1.38 & $€ 2826$ \\
\hline 6 & $\mathrm{H} 41 \mathrm{X}$ & $\begin{array}{l}\text { Malignancy of hepatobiliary system, pan- } \\
\text { creas W/O catastrophic CC }\end{array}$ & 4 & $€ 792$ & $€ 2045$ & 0.66 & $€ 1350$ \\
\hline 7 & $\mathrm{H} 41 \mathrm{M}$ & $\begin{array}{l}\text { Malignancy of hepatobiliary system, pan- } \\
\text { creas W catastrophic CC }\end{array}$ & 9 & $€ 1754$ & $€ 2045$ & 1.60 & $€ 3266$ \\
\hline 8 & $\Sigma 22 \mathrm{X}$ & Other neoplastic disorders W/O CC & 2 & $€ 638$ & $€ 2045$ & 0.50 & $€ 1020$ \\
\hline 9 & $\Pi 40 \mathrm{M}$ & Digestive malignancy $\mathrm{W}$ catastrophic $\mathrm{CC}$ & 10 & $€ 1100$ & $€ 2045$ & 2.43 & $€ 4971$ \\
\hline 10 & A31M & Respiratory neoplasms W catastrophic CC & 9 & $€ 1634$ & $€ 2045$ & 2.14 & $€ 4369$ \\
\hline 11 & $\Upsilon 22 \mathrm{M}$ & $\begin{array}{l}\text { Kidney and urinary tract neoplasms W } \\
\text { catastrophic or severe CC }\end{array}$ & 7 & $€ 1373$ & $€ 2045$ & 2.28 & $€ 4672$ \\
\hline 12 & $\Sigma 01 \mathrm{M}$ & $\begin{array}{l}\text { Lymphoma and leukaemia } \mathrm{W} \text { major } \mathrm{OR} \\
\text { procedures } \mathrm{W} \text { catastrophic or severe } \mathrm{CC}\end{array}$ & 23 & $€ 8000$ & $€ 2045$ & 6.25 & $€ 12,790$ \\
\hline 13 & Г20X & $\begin{array}{l}\text { Malignancy, female reproductive system } \\
\text { W/O catastrophic CC }\end{array}$ & 4 & $€ 1100$ & $€ 2045$ & 0.90 & $€ 1843$ \\
\hline
\end{tabular}

$C C$ Catastrophic or Severe CC, $D R G$ diagnosis-related group, $K E N-D R G$ Greek diagnosis-related group, $L O S$ length of stay, $R / N$ row number, $W$ with, $W / O$ without 
Table 4 Prediction model of DRG price based on the examined KEN-DRG: Proposal B

\begin{tabular}{|c|c|c|c|c|c|c|c|c|c|}
\hline 1 & 2 & 3 & 4 & 5 & 6 & 7 & 8 & 9 & 10 \\
\hline $\mathrm{R} / \mathrm{N}$ & $\begin{array}{l}\text { KEN-DRG } \\
\text { code }\end{array}$ & $\begin{array}{l}\text { AR-DRG } \\
\text { code }\end{array}$ & KEN-DRG description & $\begin{array}{l}\text { LOS by KEN- } \\
\text { DRG (days) }\end{array}$ & $\begin{array}{l}\text { KEN-DRG } \\
\text { price }\end{array}$ & $\begin{array}{l}\text { Real cost } \\
\text { based on } \\
\text { ALOS }\end{array}$ & $\begin{array}{l}\text { Base } \\
\text { price }\end{array}$ & $\begin{array}{l}\text { AR-DRG rela- } \\
\text { tive weights }\end{array}$ & $\begin{array}{l}\text { Proposed } \\
\text { DRG price }\end{array}$ \\
\hline 1 & $\Pi 40 \mathrm{X}$ & G60B & $\begin{array}{l}\text { Digestive malignancy W/O cata- } \\
\text { strophic CC }\end{array}$ & 3 & $€ 600$ & $€ 1520$ & $€ 2045$ & 0.87 & $€ 1779$ \\
\hline 2 & $\Sigma 20 \mathrm{M}$ & R60A & Acute leukaemia W catastrophic CC & 17 & $€ 5707$ & $€ 11,225$ & $€ 2045$ & 7.1 & $€ 14,520$ \\
\hline 3 & $\Sigma 21 \mathrm{M}$ & R61A & $\begin{array}{l}\text { Lymphoma and non-acute leukaemia } \\
\text { W catastrophic CC }\end{array}$ & 14 & $€ 3100$ & $€ 6971$ & $€ 2045$ & 4.86 & $€ 9939$ \\
\hline 4 & $\Sigma 21 \mathrm{X}$ & R61B & $\begin{array}{l}\text { Lymphoma and non-acute leukaemia } \\
\text { W/O catastrophic CC }\end{array}$ & 5 & $€ 1410$ & $€ 1462$ & $€ 2045$ & 1.83 & $€ 3742$ \\
\hline 5 & A31X & E71B & $\begin{array}{l}\text { Respiratory neoplasms W/O cata- } \\
\text { strophic CC }\end{array}$ & 5 & $€ 828$ & $€ 2826$ & $€ 2045$ & 1.17 & $€ 2393$ \\
\hline 6 & $\mathrm{H} 41 \mathrm{X}$ & H61B & $\begin{array}{l}\text { Malignancy of hepatobiliary system, } \\
\text { pancreas W/O catastrophic CC }\end{array}$ & 4 & $€ 792$ & $€ 1350$ & $€ 2045$ & 1.12 & $€ 2290$ \\
\hline 7 & $\mathrm{H} 41 \mathrm{M}$ & H61A & $\begin{array}{l}\text { Malignancy of hepatobiliary system, } \\
\text { pancreas W catastrophic CC }\end{array}$ & 9 & $€ 1754$ & $€ 3266$ & $€ 2045$ & 2.48 & $€ 5072$ \\
\hline 8 & $\Sigma 22 \mathrm{X}$ & R62B & Other neoplastic disorders W/O CC & 2 & $€ 638$ & $€ 1020$ & $€ 2045$ & 0.82 & $€ 1677$ \\
\hline 9 & П40M & G60A & $\begin{array}{l}\text { Digestive malignancy W cata- } \\
\text { strophic CC }\end{array}$ & 10 & $€ 1100$ & $€ 4971$ & $€ 2045$ & 2.15 & $€ 4397$ \\
\hline 10 & $\mathrm{~A} 31 \mathrm{M}$ & E71A & $\begin{array}{l}\text { Respiratory neoplasms W cata- } \\
\text { strophic CC }\end{array}$ & 9 & $€ 1634$ & $€ 4369$ & $€ 2045$ & 2.31 & $€ 4724$ \\
\hline 11 & $\Upsilon 22 \mathrm{M}$ & L62A & $\begin{array}{l}\text { Kidney and urinary tract neoplasms } \\
\text { W catastrophic or severe CC }\end{array}$ & 7 & $€ 1373$ & $€ 4672$ & $€ 2045$ & 1.89 & $€ 3865$ \\
\hline 12 & $\Sigma 01 \mathrm{M}$ & R01A & $\begin{array}{l}\text { Lymphoma and leukaemia } \mathrm{W} \text { major } \\
\text { OR procedures } \mathrm{W} \text { catastrophic or } \\
\text { severe CC }\end{array}$ & 23 & $€ 8000$ & $€ 12,790$ & $€ 2045$ & 9.96 & $€ 20,369$ \\
\hline 13 & Г20X & N60B & $\begin{array}{c}\text { Malignancy, female reproductive } \\
\text { system W/O catastrophic CC }\end{array}$ & 4 & $€ 1100$ & $€ 1843$ & $€ 2045$ & 0.97 & $€ 1984$ \\
\hline
\end{tabular}

$A L O S$ average length of stay, $A R-D R G$ Australian DRG system, $C C$ Catastrophic or Severe CC, $D R G$ diagnosis-related group, KEN-DRG Greek diagnosis-related group, $L O S$ length of stay, $R / N$ row number, $W$ with, $W / O$ without

\section{Discussion}

Reforming Greece's health care system through quick implementation of a DRG reimbursement system was an attempt by the Greek government to increase the transparency of the health care system, reduce its financial deficit, and to gradually implement global budgets in public hospitals. The Greek economic crisis and ensuing pressure from European financial authorities obliged Greece to implement the Greek DRG system within a very short period of time. The ARDRG classification system was chosen under the assumption that the relative resource consumptions of the Australian and Greek health care systems would be comparable. As a result, KEN-DRG pricing was not based on actual costs of hospitalization incidents and clinical protocols, but was created through a combination of cost data from the analysis of cases from selected Greek public hospitals, collected over a short period of time, and the cost-weighted coefficients of the ARDRG system of Australia. Moreover, hospital payroll costs were not included in KEN-DRG prices, although their inclusion in hospital budgets is considered important for reasons of improved financial and administrative efficiency [4, 13].
In Greece, the lack of a permanent mechanism for monitoring all hospital incidents makes analysis of the data from the KEN-DRG implementation difficult when reviewing both the prices and the ALOS. Such a quantitative analytical approach implies the existence of an advanced costing system with cost centers at the patient level, which is also currently unavailable in Greek public hospitals [10].

A limitation of our costing approach is that it provided less scope for obtaining more costing details at the patient level. Since the cost-estimate procedure was top-down and was based on the operating costs of two Internal Medicine Departments, this method overlooked a lot of lower-level details related to the resource consumption per patient, such as hours of nursing, medication, etc. Moreover, we have to consider that, depending on their type, size or specialization, there are quite a lot of differences between hospitals in terms of resource consumption for patients whose cases are classified within the same KEN-DRG code. This study is also limited by the fact that it focused on the cost calculation for a subset of KEN-DRGs and the conclusions may not be generally applicable to the implementation of the KEN-DRG system in Greece. 
Nevertheless, given the above limitations, the current study revealed significant payment disparities between the actual costs of hospitalization and the hospital reimbursement amount. Furthermore, the increased severity of illness and the higher complexity of selected cases appear to have a negative impact on the average hospital reimbursement amount, according to the KEN-DRG reimbursement system. The cost estimation analysis of selected groups of patients exposed the gap between the actual hospitalization cost and the amount of the hospital reimbursement in these cases.

In this study, two proposed models of KEN-DRG price recalculation were developed, based on the average estimated cost of hospitalization for the sample incidents. These two models appear to be more effective in the calculation of cancer KEN-DRG prices in terms of the actual hospitalization cost, and could therefore improve the existing reimbursement system of Greek hospitals in the medium term.

In summary, taking into consideration that the funding of public hospitals still comes from the state budget, and global budgets are not yet available in public hospitals, we can conclude that it is crucial for the Greek government to implement an effective and more transparent DRG payment system, ensuring a fair and sustainable financing mechanism for hospitals. On the other hand, the broad picture presented in the Organisation for Economic Co-operation and Development (OECD) health policy report for Greece shows that between 2009 and 2013 there has been a reduction in health care spending by more than one-third. The reduction in the financial deficit of the health care system cannot be explained only by the implementation of the KEN-DRG system since the reduction refers to overall health expenditure [12].

All the above highlight that the current plan of the Greek government for health care system reform must be more effectively integrated and better designed in order to fulfill its promises [14].

\section{Conclusions}

With regard to the costing of cancer KEN-DRG codes, the present study shows that the current prices specified by oncological KEN-DRGs often do not match the actual average costs of hospitalization. The results also reveal that the ALOS of KEN-DRG codes does not reflect the actual ALOS of the examined sample of cases.

In terms of the existing KEN-DRG reimbursement system, the current study proposes the use of a DRG price calculation model that consists of a relative weighting factor and a base price based on a real cost calculation process on an annual basis.

These results refer to a relatively small sample of patients from a public hospital. It is therefore necessary for the Greek
Ministry of Health to define an extensive network of hospitals that will automatically provide their financial data to an organization that will collect, manage and analyze the data statistically. The purpose of this organization will be to propose, on a yearly basis, modifications to the KEN-DRG prices and ALOS, according to National Hospital Cost Data collected during the previous year.

By following approaches similar to the above-described proposed models, it would be possible, with the use of suitable cost accounting methods in large public hospitals, to analyze the cost of a greater number of homogeneous hospitalization incidents in order to calculate with accuracy a base price and relative weights for all existing KEN-DRGs.

In conclusion, the launch of the Greek DRG system triggered the need for further research in order for the DRG model to fulfill its promises. In addition, it should be stressed that the present study, as well as other related studies, make it possible to know the actual cost of hospitalization, and can contribute to the creation of a cost database over time at the level of hospitals or specific clinical departments.

Data Availability Statement The data used to support the findings of this paper were part of a $\mathrm{PhD}$ thesis written in the Greek language, but have not yet been made accessible. The data will be available approximately 1 year after publication upon application to the website of the Greek National Archive of PhD Theses (EADD; https://www.didaktorika.gr/ eadd/).

Author Contributions All authors contributed equally to the design and implementation of the research, analysis of the results and writing of the manuscript. In addition, all authors read and approved the final version of the manuscript.

\section{Compliance with Ethical Standards}

Conflicts of interest Panos Panagiotopoulos, Nikolaos Maniadakis, George Papatheodoridis and Dimitris Pektasidis declare they have no conflicts of interest with respect to the contents of this article.

Funding The authors received no specific funding for this study.

Open Access This article is distributed under the terms of the Creative Commons Attribution-NonCommercial 4.0 International License (http://creativecommons.org/licenses/by-nc/4.0/), which permits any noncommercial use, distribution, and reproduction in any medium, provided you give appropriate credit to the original author(s) and the source, provide a link to the Creative Commons license, and indicate if changes were made.

\section{References}

1. Busse R, Blümel M. Germany: health system review. Health Syst Trans. 2014;16(2):1-296. 
2. Busse R, Geissler A, Aaviksoo A, Cots F, Häkkinen U, Kobel $\mathrm{C}$, et al. Diagnosis related groups in Europe: moving towards transparency, efficiency, and quality in hospitals? BMJ. 2013;346:f3197.

3. Busse R, Riesberg A. Health Care Systems in Transition: Germany. Copenhagen: WHO Regional Office for Europe, on behalf of the European Observatory, on behalf of the European Observatory on Health Systems and Policies; 2004.

4. Economou C, Kaitelidou D, Kentikelenis A, Sissouras A, Maresso A. The Impact of the financial crisis on health and the health system in Greece. WHO/European Observatory on Health Systems and Policies Case Study; 2014.

5. Economou C. Greece: health system review. Health Syst Trans. 2010;12(7):1-180.

6. Fetter R, Brand D, Gamache D. DRGs: their design and development. Chicago: Health Administration Press; 2000.

7. Geissler A, Quentin W, Scheller-Kreinsen D, Busse R. Introduction to DRGs in Europe: common objectives across different hospital systems. In: Busse R, Geissler A, Quentin W, Wiley MM, editors. Diagnosis related groups in europe: moving towards transparency, efficiency and quality in hospitals?. Maidenhead: Open University Press; 2011.

8. Hellenic Ministry of Health and Social Solidarity. KEN-DRG Committes' Reports. 2011. http://www.moh.gov.gr/articles/health/ domes-kai-draseis-gia-thn-ygeia/articles/ken-eswteriko/710-ypoyr gikes-apofaseis-egkyklioi-g-gaseis-egkyklioi-g-g. Accessed 8 Nov 2016.

9. Magnussen J, Solstad K. Case-based hospital financing: the case of Norway. Health Policy. 1994;28:23-36.

10. National School of Public Health Athens. DRG Implementation Mechanism in Greece. 2011. Available at: http://www.esdy.edu. gr/files/009_Oikonomikon_Ygeias/\%CE\%9C\%CE\%B5\%CE\%B8 \%СЕ\%BF\%СЕ\%В4\%СЕ\%BF\%СЕ\%ВB\%СЕ\%ВF\%СЕ\%В3\%СЕ $\% \mathrm{AF} \% \mathrm{CE} \% \mathrm{~B} 1 \% 20 \% \mathrm{CF} \% 80 \% \mathrm{CF} \% 81 \% \mathrm{CE} \% \mathrm{BF} \% \mathrm{CF} \% 83 \% \mathrm{CE} \% \mathrm{~B}$ $1 \% \mathrm{CF} \% 81 \% \mathrm{CE} \% \mathrm{BC} \% \mathrm{CE} \% \mathrm{BF} \% \mathrm{CE} \% \mathrm{~B} 3 \% \mathrm{CE} \% \mathrm{AE} \% \mathrm{CF} \% 82 \% 20$ \%CE\%9A\%CE\%95\%CE\%9D-DRGs_final.pdf. Accessed 8 Nov 2016.

11. OECD health policy in Greece. http://www.oecd.org/greece/Healt h-Policy-in-Greece-January-2016.pdf. Accessed 8 Nov 2016.

12. Polyzos N, et al. Reforming reimbursement of public hospitals in Greece during the economic crisis: implementation of a DRG system. Health Policy. 2013;109:14-22.

13. Xenos $\mathrm{P}$, et al. Modern methods of hospital funding, competition and financial incentives. Arch Hellen Med. 2014;31:172-85.

14. Xenos P, Yfantopoulos J, Nektarios M, Polyzos N, Tinios P, Constantopoulos A. Efficiency and productivity assessment of public hospitals in Greece during the crisis period 2009-2012. Cost Eff Resour Alloc. 2017;15:6

15. Yfantopoulos J. Health economics, theory and policy [in Greek]. 1st ed. Athens: Typothito; 2006. 